\title{
Neutrino and antineutrino quasielastic interactions with nuclei
}

\author{
M. Martini, ${ }^{1,}{ }^{*}$ M. Ericson, ${ }^{2,3}$ G. Chanfray, ${ }^{2}$ and J. Marteau ${ }^{2}$ \\ ${ }^{1}$ CEA/DAM/DIF, F-91297 Arpajon, France \\ ${ }^{2}$ Université de Lyon, Univ. Lyon 1, CNRS/IN2P3, IPN Lyon, F-69622 Villeurbanne Cedex, France \\ ${ }^{3}$ Theory Group, Physics Department, CERN, CH-1211 Geneva, Switzerland
}

(Received 24 February 2010; published 23 April 2010)

\begin{abstract}
We investigate the interaction of neutrinos and antineutrinos with nuclei. We explore, in particular, the role played by multinucleon excitations, which can contaminate the quasielastic cross section. For neutrinos the multinucleon term produces a sizable increase in the quasielastic cross section. Part of the effect arises from tensor correlations. For antineutrinos this influence is smaller, owing to the axial-vector interference, which increases the relative importance of the terms that are not affected by these multinucleon excitations.
\end{abstract}

DOI: 10.1103/PhysRevC.81.045502

PACS number(s): 25.30.Pt, 13.15.+g, 24.10.Cn

\section{INTRODUCTION}

The progress in neutrino experimental physics has allowed the measurement of several partial cross sections in the interaction with nuclei. Most data concern ratios of cross sections [1-7] but some absolute values are now available [8-10]. In particular, the MiniBooNE collaboration has measured the neutrino's charged-current $(\mathrm{CC})$ quasielastic $(\mathrm{QE})$ cross section on ${ }^{12} \mathrm{C}$ for a neutrino beam with an average energy of $788 \mathrm{MeV}$ [10]. In this work ejected nucleons are not detected and the QE cross section is defined as the one for processes in which only a muon is detected in the final state. However, it is possible that in the neutrino interaction a pion produced via the excitation of the $\Delta$ resonance escapes detection, for instance, because it is reabsorbed in the nucleus, leading to multinucleon emission. In this case it simulates a $\mathrm{QE}$ process. The MiniBooNE analysis of the data corrects for this possibility via a Monte Carlo evaluation of this process. The net effect amounts to a reduction in the observed QE cross section. After application of this correction the $\mathrm{QE}$ cross section thus defined still displays an anomaly compared to a relativistic Fermi gas prediction. The prediction, which is sensitive to the cutoff mass of the axial form factor, fits the data provided a modified axial form factor is introduced in the calculation, with an increase in the axial cutoff mass from the accepted value $M_{A}=1.03 \mathrm{GeV}$ to the value $M_{A}=1.35$ $\mathrm{GeV}$; otherwise the calculated cross section is too small [10].

On the theoretical side, Martini et al. [11] have drawn attention to the existence of additional sources of multinucleon emission that are susceptible to produce an apparent increase in the "QE" cross section. Their evaluation, although approximate, of this contribution shows that it is able to account for this apparent increase. It stresses, in particular, the role played by the $N N$ tensor correlations in this enhancement. In clarifying this point we are naturally led to explore the corresponding effect in the case of antineutrinos. We show that antineutrinos can provide a useful test of the origin of this anomaly, which is the aim of the present work.

\footnotetext{
*marco.martini@ceafr
}

\section{MODEL}

In our approach, the same as that in Ref. [11], the neutrino cross section on nuclei is expressed in terms of the nuclear response functions treated in the random phase approximation (RPA). The only nucleon resonance taken into account is the $\Delta$ one. Several responses enter this interaction, as exemplified here in a simplified expression of the $\mathrm{CC}$ cross section where the lepton mass is ignored and the $\Delta$ width is taken to 0 . It reads

$$
\begin{aligned}
\frac{\partial^{2} \sigma}{\partial \Omega \partial k^{\prime}}= & \frac{G_{F}^{2} \cos ^{2} \theta_{c}\left(\boldsymbol{k}^{\prime}\right)^{2}}{2 \pi^{2}} \cos ^{2} \frac{\theta}{2}\left\{G_{E}^{2}\left(\frac{q_{\mu}^{2}}{\boldsymbol{q}^{2}}\right)^{2} R_{\tau}^{N N}\right. \\
& +G_{A}^{2} \frac{\left(M_{\Delta}-M\right)^{2}}{2 \boldsymbol{q}^{2}} R_{\sigma \tau(L)}^{N \Delta}+G_{A}^{2} \frac{\left(M_{\Delta}-M\right)^{2}}{\boldsymbol{q}^{2}} \\
& \times R_{\sigma \tau(L)}^{\Delta \Delta}+\left(G_{M}^{2} \frac{\omega^{2}}{\boldsymbol{q}^{2}}+G_{A}^{2}\right)\left(-\frac{q_{\mu}^{2}}{\boldsymbol{q}^{2}}+2 \tan ^{2} \frac{\theta}{2}\right) \\
& \times\left[R_{\sigma \tau(T)}^{N N}+2 R_{\sigma \tau(T)}^{N \Delta}+R_{\sigma \tau(T)}^{\Delta \Delta}\right] \pm 2 G_{A} G_{M} \frac{k+k^{\prime}}{M} \\
& \left.\times \tan ^{2} \frac{\theta}{2}\left[R_{\sigma \tau(T)}^{N N}+2 R_{\sigma \tau(T)}^{N \Delta}+R_{\sigma \tau(T)}^{\Delta \Delta}\right]\right\},
\end{aligned}
$$

where $G_{F}$ is the weak coupling constant, $\theta_{c}$ the Cabbibo angle, $k$ and $k^{\prime}$ the initial and final lepton momenta, $q_{\mu}=k_{\mu}-k_{\mu}^{\prime}=(\omega, \boldsymbol{q})$ the four-momentum transferred to the nucleus, $\theta$ the scattering angle, and $M_{\Delta}(M)$ the $\Delta$ (nucleon) mass. The plus (minus) sign in Eq. (1) stands for the neutrino (antineutrino) case. The existence of this axial-vector interference term is crucial in the present work. The various responses are related to the imaginary part of the corresponding full polarization propagators:

$$
R(\omega, q)=-\frac{\mathcal{V}}{\pi} \operatorname{Im}[\Pi(\omega, q, q)] .
$$

They are related to the inelastic cross section for a given coupling. For instance, for the isospin operator,

$$
\begin{aligned}
R_{\tau}= & \sum_{n}\left\langle n\left|\sum_{j=1}^{A} \tau(j) e^{i \boldsymbol{q} \cdot \boldsymbol{x}_{j}}\right| 0\right\rangle \\
& \times\left\langle n\left|\sum_{k=1}^{A} \tau(k) e^{i \boldsymbol{q} \cdot \boldsymbol{x}_{k}}\right| 0\right\rangle^{*} \delta\left(\omega-E_{n}+E_{0}\right) .
\end{aligned}
$$




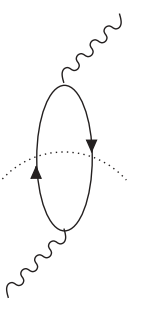

(a)

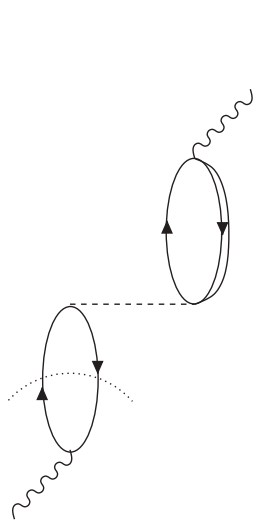

(b)

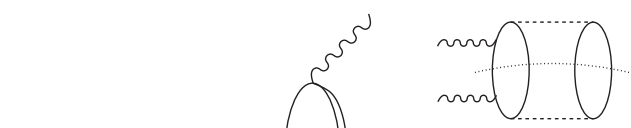

(a)

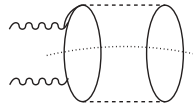

(b)

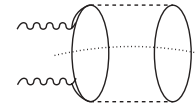

(c)

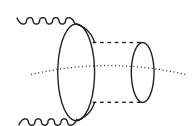

(d)

FIG. 2. Examples of contributions to the two-nucleon ejection term from (a) $R^{N N}$, (b) $R^{N \Delta}$, and (c, d) $R^{\Delta \Delta}$. Diagram (d) represents an example of a modification of the $\Delta$ width in the medium.

The collective effects produce a mild suppression of the $\mathrm{QE}$ response owing to the repulsive nature of the residual interaction. The effect that we want to discuss here is of a different nature. It concerns the multinucleon $(n \mathrm{p}-n \mathrm{~h})$ ejection in neutrino interactions other than that owing to the final-state interaction. Here several sources of multinucleon emission enter our description and all types of responses, $R^{N N}, R^{N \Delta}$, and $R^{\Delta \Delta}$, contribute. Examples are given in Fig. 2. One of these multinucleon sources arises from the modification of the $\Delta$ width in the nuclear medium [Fig. 2(d)]. This effect was introduced and parametrized by Oset and Salcedo [12] in the case of real pion or photon absorption. We have used their parametrization of the modified width, although the kinematics of neutrino interaction is different, as we are in the spacelike region, which could be a source of uncertainty. For the other terms not reducible to a modification of a $\Delta$ width, of which some examples are shown in Fig. 2, we have used a parametrization of Delorme and Guichon [13]. They exploited a calculation by Shimizu and Faessler [14] of the absorptive part of the $p$-wave pion-nucleus optical potential at threshold, which writes $\left(4 \pi / 2 m_{\pi}\right) \vec{\nabla} \cdot \operatorname{Im} C_{0} \rho^{2} \vec{\nabla}$ [15]. It is known that the absorption mechanism of pions is a two-nucleon one, which means that in the final state two nucleons are ejected. In the many-body language this is a two particles-two holes (2p-2h) excitation. In Ref. [14] the absorption is described by three types of terms (see Fig. 2). The first one [Fig. 2(a), with pion lines replacing the weak current ones as in the next diagrams] arises from the nucleon-nucleon correlations, essentially from the tensor correlations. The second one involves a $\Delta$ excitation [Fig. 2(c)]. The third is an interference between the nucleon correlation and the $\Delta$ terms as in Fig. 2(b). In these graphs the coupling of the pion to the nucleon involves the pion momentum and is of the spin isospin type, $\vec{\sigma} \cdot \vec{q} \vec{\tau}$, for the nucleon, with a similar expression for $\Delta$ excitation. In the optical potential the pion momentum is expressed by the gradient; the remaining part of the optical potential with the parameter $\operatorname{Im} C_{0}$ then provides the nuclear $2 \mathrm{p}-2 \mathrm{~h}$ bare responses to a probe that couples to the spin and isospin of the nucleon. In principle it is a spin longitudinal coupling, but as this term represents a short-range effect, there is no difference in the bare case (i.e., before the RPA chain) between longitudinal and transverse spin couplings. Therefore it applies to the bare magnetic part of the vector current and to both the spin-transverse and the spin-longitudinal parts of the axial current. The pion absorption calculation in Ref. [14] is performed for a threshold pion, that is, for a vanishing three-momentum and an energy $\omega=m_{\pi}$, which does not correspond to the neutrino situation. Delorme et al. [13] then introduced in each absorption graph the corresponding energy dependence, to obtain the bare $2 \mathrm{p}-2 \mathrm{~h}$ responses to be inserted 
in the RPA chain needed for neutrino interaction. However they completely ignored the momentum dependence. They left apart the graph in Fig. 2(d), as it corresponds to a modification of the $\Delta$ width, which is taken into account separately through the parametrization of Oset et al. [12]. In the first approach we have used the procedure of Delorme $e t$ al. as such, to evaluate the bare $2 \mathrm{p}-2 \mathrm{~h}$ components to be inserted in the RPA chain for evaluation of the neutrino cross section. In our resulting $2 \mathrm{p}-2 \mathrm{~h}$ cross section the modification of the $\Delta$ width is not a dominant effect; it adds a small $2 \mathrm{p}-2 \mathrm{~h}$ component (plus a $3 \mathrm{p}-3 \mathrm{~h}$ one). It turned out that our overall multinucleon contribution, when added to the genuine QE cross section, was able to account for the anomaly without modification of the axial cutoff mass as already reported in Ref. [11].

Because our parametrization of the $2 \mathrm{p}-2 \mathrm{~h}$ piece from the extrapolation of pion absorption is questionable, as it ignores, in particular, any momentum dependence, we have also investigated the effect with our second parametrization [11] of the $2 \mathrm{p}-2 \mathrm{~h}$ contribution beyond the one that is reducible to a modification of the $\Delta$ width. In the second approach we have used a microscopic calculation by Alberico et al. [16] specifically aimed at the evaluation of the $2 p-2 h$ contribution to the isospin spin-transverse response, measured in inclusive $\left(e, e^{\prime}\right)$ scattering. Their basic graphs are similar to those of Shimizu and Faessler. In principle this method of evaluation is definitely more satisfactory, as the kinematical variables are correctly incorporated, but the results in Ref. [16] are available only for a limited set of energy and momenta. We have extended this range to cover the neutrino one through an approximate extrapolation. We refer to Ref. [11] for the details. The result was that the distribution in the energy transfer $\omega$ of the differential neutrino cross section is largely modified with a more realistic distribution, but once integrated in energy the difference is small. The corresponding neutrino and antineutrino total "QE" cross sections are practically unchanged.

\section{RESULTS}

From the way in which this $2 \mathrm{p}-2 \mathrm{~h}$ contribution is built, the corresponding coupling of the weak current to the nucleon or $\Delta$ is a spin isospin one. It is then clear that this $2 \mathrm{p}-2 \mathrm{~h}$ term only affects the magnetic and axial responses that enter the neutrino cross section. In expression (1) these are the terms in $G_{A}^{2}$ and $G_{M}^{2}$ and the interference term in $G_{A} G_{M}$. The isovector response (term in $R_{\tau}$ ) instead is not affected. This difference is the basis for the test that we propose to help elucidate the origin of the anomaly. The principle is simple. For antineutrinos the interference term in $G_{M} G_{A}$ produces a suppression of the spin isospin response contribution, while it enhances it for neutrinos. In the neutrino case the contribution from the spin isospin terms largely dominates the isovector one. If the suppression of the spin isospin part for antineutrinos by the interference term modifies the balance in such a way that the role of the isovector response in the cross section becomes appreciable, the relative role of the $2 \mathrm{p}-2 \mathrm{~h}$ part will be smaller for antineutrinos. We show here that this is indeed the case. Figure 3 displays the various components of the genuine QE

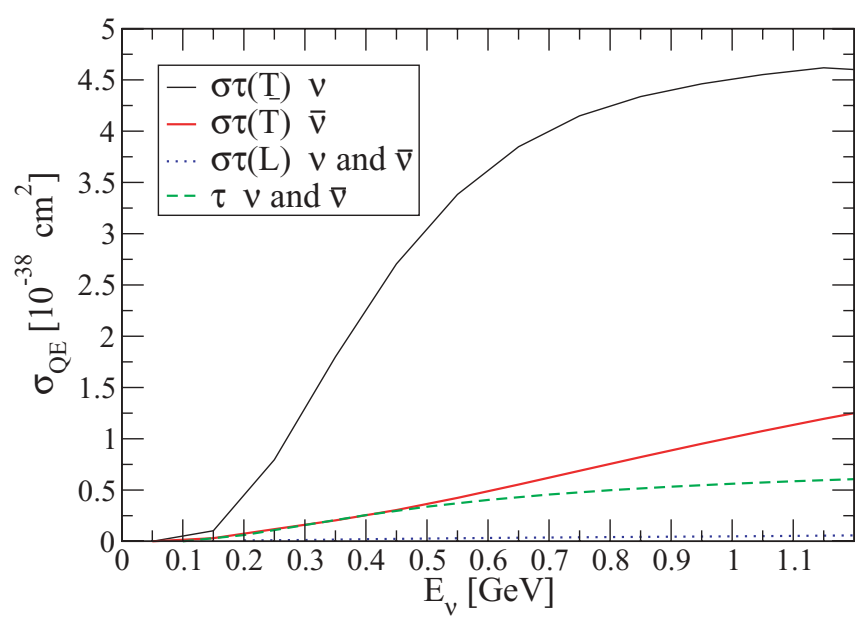

FIG. 3. (Color online) Various response contributions to the $v_{\mu^{-}}$and $\bar{v}_{\mu^{-}}{ }^{12} \mathrm{C}$ charged-current genuine quasielastic cross section. Isovector $(\tau)$ and isospin spin-longitudinal $[\sigma \tau(L)]$ components are identical for $v_{\mu}$ and $\bar{v}_{\mu}$.

(single nucleon ejection) cross section on ${ }^{12} \mathrm{C}$ as a function of the energy both for muon neutrinos and antineutrinos. The isovector part is identical in the two cases, as well as the isospin spin-longitudinal one. The isospin spin-transverse one instead changes considerably between neutrinos and antineutrinos owing to the interference term, with an appreciable reduction in the antineutrino case. As a consequence the isovector relative contribution to the total cross section becomes quite significant for antineutrinos, which opens the possibility of an experimental test. Such details are visible in Fig. 4, where the differential cross section $d \sigma / d \omega$ at $E_{v}=700 \mathrm{MeV}$ is shown in the bare and RPA case, for both neutrinos and antineutrinos. The same figure displays the axial-vector interference term, as well as the isovector component, illustrating the importance of the latter in the antineutrino case. A remark on the collective nature is in order at this stage. One notices the suppression produced by the RPA, which follows from the repulsive character of the residual interaction. It is more pronounced at low energies. The interference term, which spreads over the entire energy range, is therefore less affected by the RPA. The antineutrino cross section, which peaks at low energies, is instead very sensitive to the suppression by the collective RPA effects. Once integrated over the energy transfer, the reduction at a typical energy $E_{v}=700 \mathrm{MeV}$ is somewhat larger for antineutrinos than for neutrinos. The comparison between the two is affected by this difference. This could lead to a source of uncertainty in the comparison, as the RPA effects, which depend on the residual interaction, have not been tested in this momentum regime. However, we show here that it does not prevent the comparison from being significant for our purpose.

We now turn to the generalized "QE" cross section which includes the multinucleon contribution. The neutrino and antineutrino genuine and generalized "QE" cross sections are plotted in Fig. 5 both in the bare and RPA case. As was already discussed in Ref. [11] the agreement with the MiniBooNE experimental neutrino data $[8,10]$ is better when the $n \mathrm{p}-n \mathrm{~h}$ component is added to the genuine $\mathrm{QE}$ cross section, whether 


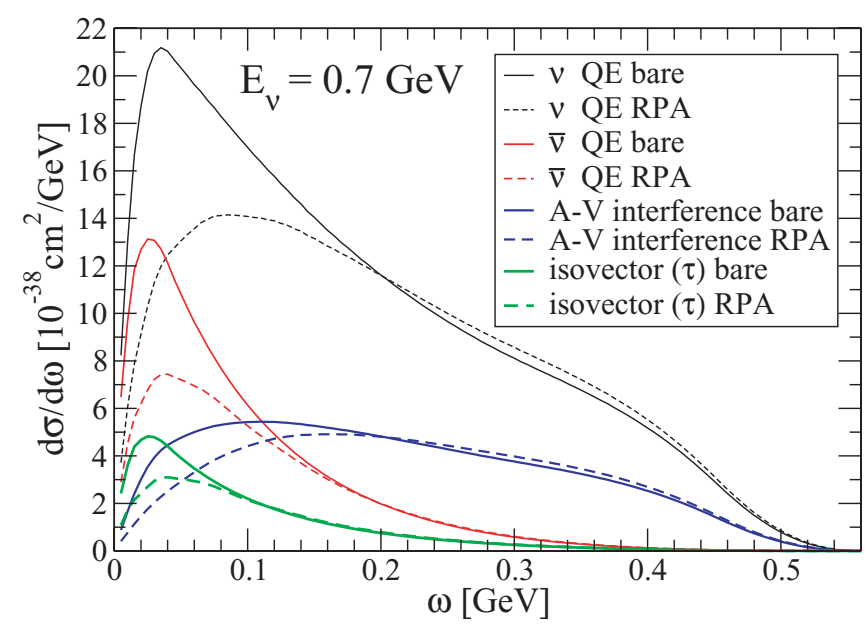

FIG. 4. (Color online) Differential CC $v_{\mu^{-}}$and $\bar{v}_{\mu^{-}}{ }^{12} \mathrm{C}$ cross sections versus energy transfer.

in the free or in the RPA case. Our prediction for the generalized neutrino "QE" cross section shows only a moderate sensitivity to the collective aspects. For antineutrinos, instead the sensitivity to RPA is somewhat greater, but it does not hide the important point that the relative importance of the $2 \mathrm{p}-2 \mathrm{~h}$ term is smaller for antineutrinos. This is illustrated in Fig. 6, which shows the ratio of the multinucleon component to the single-nucleon one, with and without RPA. In both cases, we find that the ratio for antineutrinos is reduced compared to that for neutrinos by a factor of 1.7 at $E_{v}=$ $700 \mathrm{MeV}$. To eliminate the uncertainties related to the neutrino energy reconstruction, Table I reports the values of $\mathrm{QE}$ and multinucleon cross sections on ${ }^{12} \mathrm{C}$, as well as their sum, averaged over the respective neutrino [10] and antineutrino [17] MiniBooNE fluxes, so as to provide quantities more in touch with an experimental analysis. We give these quantities in both the RPA and the free case, and various situations are possible. For instance, if our RPA description holds, the ratio
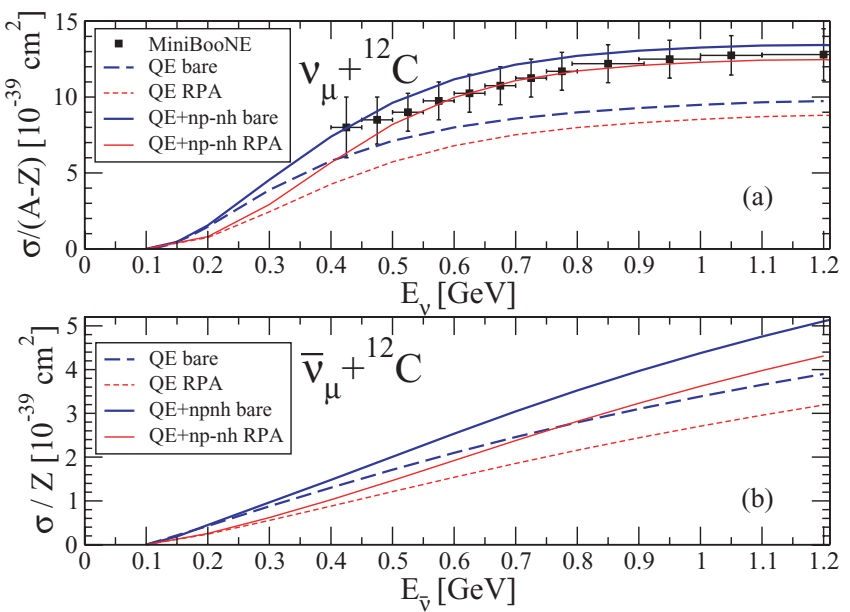

FIG. 5. (Color online) (a) $v_{\mu^{-}}{ }^{12} \mathrm{C}$ and (b) $\bar{v}_{\mu^{-}}{ }^{12} \mathrm{C} \mathrm{CC}$ "quasielastic" cross sections per neutron and per proton, with and without the multinucleon component, as a function of neutrino energy. Experimental points are taken from Ref. [10].

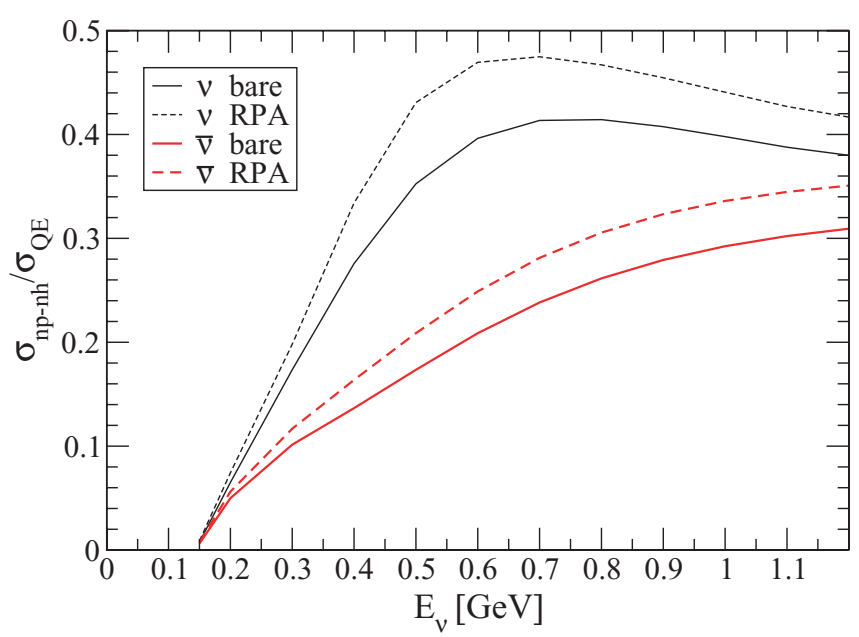

FIG. 6. (Color online) Ratio of the multinucleon component of the "quasielastic" cross section on ${ }^{12} \mathrm{C}$ to the single-nucleon one for $\nu_{\mu}$ and $\bar{v}_{\mu}$ as a function of neutrino energy.

of the generalized "QE" cross section, which is the measured cross section, to a theoretical free Fermi gas model is 1.22 for neutrinos and 0.99 for antineutrinos, significantly lower. In the extreme case where RPA effects are totally absent, the corresponding ratios are 1.37 for neutrinos and 1.25 for antineutrinos. In all cases the antineutrino numbers are smaller and the difference may be detectable, which offers an experimental test. For neutrinos the fit to the QE data in a relativistic Fermi gas description required an appreciable increase in the axial cutoff mass [10]. For antineutrinos the increase needed to account for the data in the same model should be smaller, as the relative role of multinucleon ejection is reduced. This difference offers a possible way to shed light on the origin of the anomaly. Of course the difference that occurs owing to the fact that the target is not pure carbon but $\mathrm{CH}_{2}$ affects exclusively the antineutrino cross section, reducing trivially the importance of nuclear effects. It has to be taken into account to reach a significant comparison between neutrino and antineutrino cross sections.

Finally, we would like to comment on the absence of final-state interactions in our evaluation. In addition to the absorption of a produced pion, it also ignores the possibility of an ejected nucleon interacting with the nucleus, emitting another nucleon, which leads to a final state with two nucleons ejected, the same type of final state as discussed in this

TABLE I. MiniBooNE flux-integrated CC $v_{\mu}{ }^{-12} \mathrm{C}$ and $\bar{v}_{\mu}{ }^{-12} \mathrm{C}$ total cross sections per neutron and per proton, respectively, in units of $10^{-39} \mathrm{~cm}^{2}$. The experimental CC QE $v_{\mu}{ }^{-12} \mathrm{C}$ value measured by MiniBooNE was $9.429 \times 10^{-39} \mathrm{~cm}^{2}$, with a total normalization error of $10.7 \%$ [10].

\begin{tabular}{cccccccc}
\hline \hline & \multicolumn{4}{c}{$v$} & & \multicolumn{3}{c}{$\bar{v}$} \\
\cline { 2 - 3 } \cline { 6 - 8 } & QE & $n \mathrm{p}-n \mathrm{~h}$ & $\mathrm{QE}+n \mathrm{p}-n \mathrm{~h}$ & & $\mathrm{QE}$ & $n \mathrm{p}-n \mathrm{~h}$ & $\mathrm{QE}+n \mathrm{p}-n \mathrm{~h}$ \\
\hline Bare & 7.46 & 2.77 & 10.23 & & 2.09 & 0.52 & 2.61 \\
RPA & 6.40 & 2.73 & 9.13 & & 1.60 & 0.47 & 2.07 \\
\hline \hline
\end{tabular}


work. For the final-state interaction effect all responses are concerned, not just the spin isospin ones. This was taken into account by Benhar and Meloni [18] using a spectral function that describes the single-particle dynamics in an interacting system, deduced from experimental $\left(e, e^{\prime} p\right)$ data. Their conclusion is that this inclusion cannot explain the enhancement of the measured neutrino QE cross section. Although the final states are the same $(2 \mathrm{p}-2 \mathrm{~h})$, we are dealing in the present work with a different type of correlation. These are ground-state correlations, mostly tensor ones, which affect only the spin isospin responses, producing an enhancement of the spin isospin sum rule that is reflected in an increase in the "QE" cross section. The rest is caused by the $\Delta$ excitation or the interference between the two. These also obviously belong exclusively to the spin isospin sector, which is the basis of our test, through a comparison between neutrinos and antineutrinos. A fully realistic calculation should include both the spectral function effect and the ground-state correlations or $\Delta$ ones.

\section{SUMMARY AND CONCLUSIONS}

In summary we have studied the QE neutrino and antineutrino cross section in the case of the MiniBooNE experiment, where multinucleon ejection is not distinguishable from single-nucleon production. The "QE" cross section thus defined contains a certain proportion of $2 \mathrm{p}-2 \mathrm{~h}$ and $3 \mathrm{p}-3 \mathrm{~h}$ excitations. This proportion is large for neutrinos, which may be the interpretation of the increase in the axial cutoff mass needed to describe the data in the relativistic Fermi gas. For antineutrinos we predict a smaller role of the $2 \mathrm{p}-2 \mathrm{~h}$ component. The reason is that the vector-axial interference term produces a suppression of the spin isospin response contribution to the cross section, leaving a larger role for the isovector response, which is not affected by the $2 \mathrm{p}-2 \mathrm{~h}$ component. An experimental confirmation of this difference would signal the fact that the excess cross section belongs to the spin isospin channel, thus demonstrating the role played by the tensor correlations in neutrino-nucleus interactions.

The antineutrino mode, which is actively investigated for the general problems of neutrino oscillations and $\mathrm{CP}$ violation, is also of great relevance to the understanding of neutrinonucleus interactions.
[1] S. Nakayama et al. (K2K Collaboration), Phys. Lett. B 619, 255 (2005).

[2] M. Hasegawa et al. (K2K Collaboration), Phys. Rev. Lett. 95, 252301 (2005).

[3] A. A. Aguilar-Arevalo et al. (MiniBooNE Collaboration), Phys. Lett. B 664, 41 (2008).

[4] A. Rodriguez et al. (K2K Collaboration), Phys. Rev. D 78, 032003 (2008).

[5] K. Hiraide et al. (SciBooNE Collaboration), Phys. Rev. D 78 , 112004 (2008).

[6] A. A. Aguilar-Arevalo et al. (MiniBooNE Collaboration), Phys. Rev. Lett. 103, 081801 (2009).

[7] Y. Kurimoto et al. (SciBooNE Collaboration), Phys. Rev. D 81, 033004 (2010).

[8] T. Katori (MiniBooNE Collaboration), AIP Conf. Proc. 1189, 139 (2009).

[9] A. A. Aguilar-Arevalo et al. (MiniBooNE Collaboration), Phys. Rev. D 81, 013005 (2010).

\section{ACKNOWLEDGMENTS}

We thank D. Davesne, T. Ericson, G. T. Garvey, and T. Katori for useful discussions.
[10] A. A. Aguilar-Arevalo et al. (MiniBooNE Collaboration), arXiv:1002.2680 [hep-ex].

[11] M. Martini, M. Ericson, G. Chanfray, and J. Marteau, Phys. Rev. C 80, 065501 (2009).

[12] E. Oset and L. L. Salcedo, Nucl. Phys. A 468, 631 (1987).

[13] J. Delorme and P. A. M. Guichon, in Proceedings of $10^{e}$ biennale de physique nucléaire, Aussois, France, March 6-10, 1989, LYCEN Report 8906, p. C.4.1.

[14] K. Shimizu and A. Faessler, Nucl. Phys. A 333, 495 (1980).

[15] T. E. O. Ericson and W. Weise, Pions and Nuclei, The International Series of Monographs on Physics (Clarendon, Oxford, UK, 1988), Vol. 74.

[16] W. M. Alberico, M. Ericson, and A. Molinari, Ann. Phys. 154, 356 (1984).

[17] A. A. Aguilar-Arevalo et al. (MiniBooNE Collaboration), Phys. Rev. D 79, 072002 (2009).

[18] O. Benhar and D. Meloni, Phys. Rev. D 80, 073003 (2009). 